Relations industrielles

Industrial Relations

\title{
Problèmes de formation professionnelle
}

\section{Léonce Girard}

Volume 6, numéro 4, septembre 1951

URI : https://id.erudit.org/iderudit/1023135ar

DOI : https://doi.org/10.7202/1023135ar

Aller au sommaire du numéro

\section{Éditeur(s)}

Département des relations industrielles de l'Université Laval

ISSN

0034-379X (imprimé)

1703-8138 (numérique)

Découvrir la revue

\section{Citer cet article}

Girard, L. (1951). Problèmes de formation professionnelle. Relations industrielles / Industrial Relations, 6(4), 109-114.

https://doi.org/10.7202/1023135ar
Résumé de l'article

Relèvent-ils de l'Etat ou de la profession ? - Ce qu'on en pense à Paris, Romans, Bruxelles et Rome. - Doit-on adopter une formule rigide dans le Québec ?
Tous droits réservés @ C Département des relations industrielles de l’Université Laval, 1951
Ce document est protégé par la loi sur le droit d'auteur. L’utilisation des services d'Érudit (y compris la reproduction) est assujettie à sa politique d'utilisation que vous pouvez consulter en ligne.

https://apropos.erudit.org/fr/usagers/politique-dutilisation/ 


\title{
Problèmes de formation professionnelle
}

\author{
Relevent-ils de l'Etat ou de la profession? - \\ Ce qu'on en pense à Paris, Romans, Bruxelles et \\ Rome. - Doit-on adopter une formule rigide dans \\ le Québec?
}

\author{
par LÉONCE GIRARD, \\ administrateur de la Commission d'apprentissage de la chaussure
}

Aujourd'hui, la formation professionnelle est devenue une nécessité. Le monde est dans un état perpétuel de changement. Chaque jour, apparaissent nombre de machines nouvelles. Chaque jour, on adopte des centaines de méthodes nouvelles. $\mathrm{Du}$ fait de ces inventions, les problèmes de la fabrication s'accroissent constamment, et l'employé qui veut réellement progresser et vivre de son métier doit être pourvu d'une solide formation professionnelle.

La Loi de l'Aide à l'Apprentissage, adoptée pour répondre à cette nécessité, a posé plusieurs problèmes aux représentants de l'industrie, aux sociologues et au gouvernement. Pour quelque temps, on l'a considérée, dans certains milieux, comme une révolution dans l'enseignement des métiers. Pensez donc! L'industrie et la profession qui voulaient s'occuper de formation professionnelle !

A notre point de vue, elle a eu, pour le moins, cette heureuse conséquence d'éveiller les esprits et de poser nettement ce problème: $\mathrm{A}$ qui incombe la formation professionnelle ? Est-ce que l'Etat doit en avoir la responsabilité ? N'est-ce pas plutôt le rôle de l'industrie ou de la profession de voir à la formation technique de ses employés?

Depuis cinq ans, ce problème a été considéré sur toutes ses faces, tantôt avec passion et intérêt, tantôt avec toute la sérénité que requiert l'étude de toute question de ce genre.
Ces années d'expérience nous permettent maintenant d'exprimer une opinion au moins plausible sur ce sujet d'actualité et de grand intérêt pour l'avenir des industries de notre province.

\section{LE PRINCIPE}

La Loi de l'Aide à l'Apprentissage de 1945 énonçait en principe que les groupements d'employeurs et de salariés sont véritablement intéressés dans les problèmes de l'apprentissage, de l'adaptation et de la réadaptation et que les pouvoirs publics doivent aider à les résoudre.

L'Assemblée Législative du Québec semblait, par cet énoncé, reconnaître que le rôle des pouvoirs publics consistait à aider à résoudre les problèmes et non pas à se substituer aux groupes d'employeurs et de salariés.

Ce principe semblait n'être que l'énoncé, sous une autre forme, du grand principe de sociologie chrétienne qui veut que «l'autorité publique abandonne aux groupements de rang inférieur le soin des affaires qui les concernent et remplissent ses propres fonctions de diriger, surveiller, stimuler, contenir, selon que le comportent les circonstances ou l'exigent les nécessités. »

Ici encơre, on demande à l'Etat de se borner à ses propres fonctions d'aider ou, plus précisément, de diriger, surveiller, stimuler, contenir. Cette aide, l'Etat ne doit pas l'ap- 
porter toujours de la même façon, mais bien selon les besoins et les circonstances, en autant que les intéressés sont plus ou moins aptes à régler leurs problèmes.

et pour parler de façon plus concrète, l'Etat doit aider à la formation professionnelle et à l'apprentissage d'après les besoins de chaque industrie; ici l'Etat doit diriger ou stimuler pour que l'apprentissage se développe; là, il doit surveiller et contenir pour qu'il ne se crée aucun abus.

Les délégués du gouvernement canadien, à la $3 \mathrm{e}$ conférence des $\mathrm{Na}$ tions Unies membres de l'organisation internationale du travail, en sont venus également à la conclusion que, dans chaque programme d'apprentissage, une représentation intégrale des employeurs et des travailleurs devrait être assurée, par exemple, au moyen de comités paritaires de métiers et de comités d'apprentissage dans les entreprises.

Sur les principes mêmes, il existe présentement dans la province très peu de divergences d'opinions fondamentales. Sauf de très rares exceptions, tous admettent que l'industrie devrait promouvoir la formation professionnelle et que les pouvoirs publics devraient aider. De la part de l'Etat, de la haute administration gouvernementale, des dirigeants des écoles, aucune réticence, tout au contraire la plus loyale adhésion au principe.

\section{MODALITES D'APPLICATION}

Là où les nuances d'opinion se révèlent, c'est lorsqu'il s'agit des modalités d'application. L'Etat doit aider d'après les besoins et les circonstances. Mais qu'est-ce que peut faire la profession et qu'est-ce que peut faire l'Etat?

En 1945, nous avons entendu un dirigeant de l'enseignement spécialisé, (aui n'y est plus d'ailleurs aujourd'hui) dire à un groupe d'employeurs et d'employés qui voulaient organiser une école d'apprentissage dans une industrie tres compliquée: «Nous allons organiser votre ecole, et vous allez en ètre contents. » Le rôle de l'industrie, d'après lui, consistait à ne rien faire et à prendre ce que l'Etat donnerait. L'Etat devait tout faire, seul. Entre cette opinion et celle qui veut que l'Etat ne fasse rien, il y a une marge. Et c'est cette marge que nous chercherons à établir.

Nous avons eu, en octobre dernier, le grand avantage de visiter, en Europe, plusieurs institutions de formation professionnelle. Il nous fait plaisir de nous rendre à l'invitation qu'on nous a faite de reproduire ici quelques notes prises au cours des entrevues, concernant la participation de l'Etat, de la profession, des individus à l'apprentissage et l'enseignement des métiers.

\section{Italie}

En Italie, l'enseignement relatif à la formation professionnelle est à refaire. L'Italie se relève actuellement de la guerre; le gouvernement italien n'a pas d'écoles de formation professionnelle proprement dites, sauf dans le bâtiment; toute personne qui le désire peut ouvrir une école de formation professionnelle; le gouvernement vérifie la compétence des professeurs, l'outillage, les moyens de formation et si tout est jugé satisfaisant, donne des subsides; il paiera, selon le cas, une partie ou même la totalité des dépenses. Jusqu'à date, la formation professionnelle s'est adressée aux vétérans et à ceux qui n'ont pas d'emploi; c'est l'intention du gouvernement, dans un avenir rapproché, d'ouvrir de véritables écoles de formation professionnelle. En Italie, la formation des jeunes relève du Ministère de l'Education; la formation des :-tultes, du Ministère du Travail.

Dans le bâtiment, le gouvernement a entrepris la construction de maisons ouvrières qui servent, en 
même temps, d'école d'apprentissage. Les personnes sans emploi sont tenues, sous peine d'être privées de secours, d'apprendre un métier; les chantiers sont là pour cela. Les appartements sont construits «pour le compte du gouvernement et les débours sont divisés entre les deux Ministères intéressés à la fabrication de ces maisons: le Ministère du Travail et de la Prévoyance Sociale et le Ministère des Travaux Publics. »

«Le premier paie l'instruction professionnelle, le second est chargé d'acheter les matériaux pour la construction. »

\section{France}

\section{Romans}

A Romans, France, nous avons visité particulièrement les écoles de chaussures. Romans est une ville de chaussures; en fait, la ville ne vit pratiquement que de cette industrie. Environ $90 \%$ des employés de la ville y gagnent leur subsistance. Romans, et ses alentours, compte plus de 100 fabriques de chaussures et près de 4,000 employés. Il existe deux écoles de formation professionnelle pour la chaussure, à savoir: Le Collège Technique de Romans, section de la chaussure; l'Institution de formation professionnelle de l'industrie de la chaussure.

Le Collège Technique est la propriété du Gouvernement. Le groupement social, composé des patrons et des ouvriers, n'intervient dans ce collège que de deux façons:

a) il fait des suggestions concernant l'organisation technique et l'amélioration des cours;

b) il verse, à chaque élève, pour chaque heure à l'atelier, une certaine somme, variant de 6 à 10 francs l'heure. Le but de l'industrie de la chaussure, en faisant ces versements, est de s'assurer que les élèves les plus intelligents se dirigeront vers la chaussure; avant ces versements, ces êlèves allaient plutôt du côté de l'é- lectricité ou d'autres métiers similaires.

Le collège de Romans reçoit les élèves de 14 à 18 ans, c'est-à-dire ceux qui sont tenus, de par la loi, de suivre l'enseignement professionnel. Les cours sont absolument gratuits.

Monsieur Audemart, secrétaire du syndicat de la chaussure de Romans, s'exprime comme suit: « La Commis« sion est amenée à donner son avis « ou à formuler ses suggestions sur «toutes les questions qui concernent «l'apprentissage ou la formation «professionnelle dans les Ecoles qui « existent à Romans.

«Le Collège Technique (aujour«d'hui Collège National) dépend « directement du Ministère de l'Edu«cation Nationale et le groupement «social n'a rien à voir dans sa ges«tion financière et technique, pour «lesquelles une commission spé« ciale (Conseil de Perfectionnement « Technique) a été instituée à l'éche«lon local et national.

«Toutefois, pour favoriser et dé« velopper l'apprentissage à la Sec«tion de Cordonnerie de cette école, «le Groupement Social accorde de « ses propres deniers, aux élèves ro«manais et péageois, pour chaque « heure passée à la section de la cor«donnerie, une rémunération de 6 , \& 8 ou 10 frs de l'heure, selon qu'il «s'agit d'élèves de 1ère, 2ième ou «3ième année.

«Pour mémoire, rappelons que «cette école, qui reçoit des élèves "de toute la France et même de "l'Etranger, âgés de 14 à 18 ans, «forme des apprentis, des ouvriers « et des cadres. »

L'Institution de Formation Professionnelle, 1 , ave Duchesne, Romans, ne va pas en concurrence avec le Collège Techniaue. Elle enseigne aux ouvriers, déjà employés dans l'industrie de la chaussure et qui veulent se perfectionner dans leurs opérations ou apprendre d'autres opérations. Cette école a été formée pen- 
dant l'occupation. Au début, elle était uniquement réservée aux travailleurs en chaussures de Romans. Aujourd'hui, elle reçoit des élèves, et même en majorité, venant des centres en dehors de Romans.

Cette école est encore la propriété de l'industrie. $\mathrm{Vu}$ l'affluence des élèves de l'extérieur, elle passera', dans un avenir rapproché, sous l'administration du gouvernement et l'industrie n'interviendra que pour aider. Les cours sont gratuits; l'ouvrier qui les suit reçoit, pour chaque heure de travail à l'école, le même taux de salaire qu'il reçoit à l'atelier.

Les professeurs sont payés à même un budget d'état et la subvention du groupement social alimenté par une cotisation patronale. Les professeurs, pour les cours du soir, sont des hommes d'expérience qui travaillent dans les manufactures pendant le jour.

A l'Institution, le groupement social a beaucoup plus d'influence qu'au Collège. Le groupement, composé de patrons et d'ouvriers, se rassemble souvent, fait des suggestions et aide à la direction.

«L'Institution de Formation Professionnelle de la Chaussure, agréée par le Ministère du Travail et gérée par le Groupement Social Interprofessionnel de Romans et sa région, s'adresse aux adultes et forme des ouvriers qualifiés et des cadres de maîtrise pour l'industrie de la chaussure. 》

\section{«L'Institution comprend quatre} sections:

«La Promotion Ouvrière a pour but de perfectionner la main d'oeuvre travaillant dans des établissements industriels. La demande d'entrée en stage doit être formulée par l'employeur.»

«La section du Reclassement professionnel s'adresse aux personnes désireuses de se créer une situation dans l'industrie de la chaussure, aux personnes sans emploi ou à celles obligées de changer de profession pour des raisons physiques ou économiques. Les reclassés perçoivent un salaire horaire établi par décret du ministre du Travail. Les stagiaires seront placés par l'Institution, dans la limite des demandes de l'indus. trie. »

«Le stage spécial de Promotion Ouvrière est réservé aux ouvriers empêchés de suivre les cours normaux ou les ayant déjà suivis. »

«L'Institution accepte en stage des adultes ayant terminé leurs études de culture générale et désirant s'initier rapidement à la technique de la fabrication de la chaussure. »

\section{Paris}

L'Ecole Hôtelière de Paris est la propriété de l'Association Professionnelle pour le développement de l'Enseignement Technique et professionnel dans la Corporation des Hôteliers, Restaurateurs et Limonadiers; en d'autres mots, c'est la profession, section des patrons, qui est propriétaire.

Ce genre d'école tombe sous la juridiction du Ministère de l'Education Nationale; le gouvernement a souscrit des fonds pour la construction de l'édifice et a pris une hypothèque sur l'école; l'Association pourrait, en tout temps, remettre le montant au $\mathrm{Mi}$ nistère et rester seule propriétaire.

Le syndicat ouvrier n'a aucun droit à la gestion de l'école; toutefois, des représentants du syndicat font partie du jury aux examens des élèves; des représentants du syndicat font aussi partie du conseil de perfectionnement. Ce conseil n'a pas siégé beaucoup ces derniers temps à cause de la réorganisation d'aprèsguerre; mais il sera appelé à se rassembler régulièrement.

L'école est maintenue moyennant une taxe d'apprentissage prélevée de tous les établissements qui font partie de la profession; un hôtelier, par exemple, est libre de verser sa cotisation soit à l'école, soit au percepteur de l'impôt. C'est l'Etat qui paie le personnel enseignant.

La Revue des relations industrielles 


\section{Belgique}

\section{Bruxelles}

En Belgique, la position de l'Etat et des individus par rapport à l'enseignement professionnel est resté, jusqu'à date, d'initiative privée. Ce sont les catholiques qui ont organisé des écoles et promu l'enseignement. A date, il y a des écoles d'initiative privée, des écoles communales et des écoles provineiales. C'est d'abord l'individu, la commune ou la province qui doit bâtir l'école à ses propres frais; lorsque l'école a fonctionné avec succès pendant quelque temps, le gouvernement lui donne des subsides. Ces subsides varient d'après le parti au pouvoir. Si le gouvernement est constitué par des partisans de la' gauche, ce gouvernement ne détruit pas les écoles, mais réduit les subsides au minimum. Si, par contre, le gouvernement est constitué par des éléments de droite, favorables aux catholiques, les subsides sont plus généreux. Voici, par exemple, une idée des subsides, lorsque l'école est reconnue. Le gouvernement paiera $75 \%$ du salaire des professeurs. Le $25 \%$ supplémentaire sera payé par l'individu, la commune ou la province; ou par les deux, selon le cas, et selon l'intérêt que l'on porte à l'école.

L'Institut des Arts et Métiers de Bruxelles est une école communale, c'est-à-dire qu'elle a été fondée et est soutenue par la ville de Bruxelles.

En Belgique, il n'y a pas comme en France de scolarité obligatoire de 14 à 18 ans. Quand même, un grand nombre de jeunes suivent les cours de formation professionnelle le soir. Ceci se fait par conviction. Un jeune apprenti sait fort bien qu'il n'avancera jamais s'il n'obtient pas son diplôme de l'école. Les patrons encouragent leurs employés à suivre des cours d'apprentissage.

Il y a, pour chaque école qui fait partie de l'Institut, un comité de surveillance composé des représentants des patrons et des ouvriers. $\mathrm{Ce}$ co- mité fait des suggestions pour l'amélioration de l'enseignement et aide au placement des élèves; il participe aussi aux examens de sortie.

Il est à noter que le Conseil Supérieur du Travail, en Belgique, s'occupe beaucoup de formation professionnelle; c'est là son premier rôle. Il fait des suggestions pour uniformiser les règlements approuvés par les communes et fait aussi des recommandations aux écoles de formation professionnelle.

\section{La Bouverie}

L'Ecole de la Chaussure de la' Bouverie est la propriété des communes qui font partie de la province de Hainaut, dans le sud de la Belgique. Les élèves de l'école viennent de la Bouverie, des villes et villages environnants. Les dépenses sont couvertes comme suit: le gouvernement de Belgique paie $60 \%$ du traitement des professeurs; le gouvernement provincial du Hainaut, $60 \%$ du budget; et les communes, la balance.

Les cours ne sont pas absolument gratuits, mais tout près. L'élève du jour paie 50 francs par année, soit $\$ 1.00$ et les élèves du soir, 20 frs. par année. L'Ecole est administrée par une commission administrative composée de délégués patronaux et ouvriers de la chaussure. Si, par exemple, le directeur veut faire nommer un nouveau professeur, il en fait la demande au conseil d'administration et envoie sa requête au Ministère du Travail de l'Etat qui publie un avis dans Le Moniteur, à l'effet qu'une position est ouverte et que les aspirants peuvent se présenter aux examens.

\section{Québec}

Ces quelques exemples me paraissent suffisants pour démontrer qu'il y a mille et une façons de promouvoir la formation professionnelle ou l'apprentissage dans une industrie. Tout dépend des besoins et des circonstances. 
Dans la province de Québec, depuis la mise en vigueur de la Loi de l'Aide à l'Apprentissage, la coopération entre l'industrie et l'Etat s'est établie de différentes façons.

En voici trois exemples.

L'industrie du bâtiment de Montréal a organisé sa propre école; elle a obtenu un local spacieux du gouvernement, et des octrois du ministère du Travail. L'industrie de la chaussure, par contre, a cherché surtout la coopération de l'Enseignement spécialisé et du Ministère du Bien-Etre Social; la commission d'apprentissage a payé le matériel et l'outillage et sest occupé de la partie technique de l'enseignement; le Ministère du BienEtre Social a fourni les locaux et paie les professeurs. Dans l'imprimerie, il existait déià, à Montréal, une école d'Arts Graphiques qui était la propriété du gouvernement provincial; la commission d'apprentissage n'a pas cherché à bâtir une autre école, mais est devenue, pour cette école, le conseil consultatif prévu dans la loi de l'Enseignement spécialisé; le comité paritaire fait appliquer, par décret, les règlements d'apprentissage.

Des centaines d'autres formules peuvent être adoptées. Ce qui nous semble important, ce n'est pas tant la modalité, mais le fait que l'industrie doit prendre la responsabilité de la formation professionnelle de ses membres et que l'Etat doit aider d'après les besoins et les circonstances. Les employeurs et les employés peuvent s'appliquer, plus particulièrement, au recrutement d'élèves de choix, à la désignation des machines en usage dans l'industrie, au placement des élèves, et à la mise en application d'un programme technique et même théorique conforme aux exigences de la profession. L'Etat doit viser, tout d abord, à procurer des locaux convenables, à rémunérer des professeurs de grande compétence, à procurer l'outillage et la machinerie modernes, et à fournir des conseillers expérimentés pour l'organisation et l'amélioration constante des écoles.

Chaque industrie devrait avoir une école d'apprentissage ou de formation professionnelle; les patrons et les ouvriers devraient coopérer à la formation des apprentis, à l'adaptation ou à la réadaptation, par le moyen d'une commission d'apprentissage. S'il n'existe aucune école dans l'industrie, la commission d'apprentissage doit, avec l'aide du conseiller technique du gouvernement, voir à son organisation.

Si l'Etat a déjà fondé une telle école, pourquoi la commission d'apprentissage ne deviendrait-elle pas le conseil consultatif prévu par la loi de l'Enseignement spécialisé ? Les directeurs des écoles techniques ou des écoles d'arts et métiers retireront de ces commissions d'apprentissage, non seulement des conseils précieux pour un enseignement pratique, mais encore une aide considérable pour le placement de leurs élèves.

Ce problème de la participation de l'industrie à la formation professionnelle sera encore longtemps un sujet de discussion. Je crois que c'est une excellente chose que les opinions restent partagées. De cette facon, nous pourrons nous faire des idées de plus en plus précises sur cet important problème. 